\title{
Michel MARCOCCIA, Analyser la communication
}

\section{numérique écrite}

Paris, A. Colin, coll. Icom. Série Discours et communication, 2016,

160 pages

\section{Liana Pop}

\section{OpenEdition}

\section{Journals}

Édition électronique

URL : http://journals.openedition.org/questionsdecommunication/11729

DOI : 10.4000/questionsdecommunication. 11729

ISSN : 2259-8901

\section{Éditeur}

Presses universitaires de Lorraine

\section{Édition imprimée}

Date de publication : 31 décembre 2017

Pagination : 473-474

ISBN : 9782814305076

ISSN : 1633-5961

\section{Référence électronique}

Liana Pop, « Michel marcoccia, Analyser la communication numérique écrite », Questions de communication [En ligne], 32 | 2017, mis en ligne le 31 décembre 2017, consulté le 04 janvier 2021.

URL : http://journals.openedition.org/questionsdecommunication/11729; DOI : https://doi.org/

10.4000/questionsdecommunication. 11729 
Pour autant tout, il répond à certaines des questions des parents sur les problèmes comportementaux des adolescents en donnant quelques clés.

Florence Michet

Mica, université Bordeaux-Montaigne, F-33600 florence.michet@etu.u-bordeaux-montaigne.fr

\section{Michel MARCOCCIA, Analyser la communication numérique écrite}

Paris,A. Colin, coll. Icom. Série Discours et communication, 2016, 160 pages

Voici un livre très utile pour tous les linguistes qui se posent des questions sur les nouvelles formes de communication numérique. Un livre clair, bien organisé, avec des explications et des descriptions précises de ce qu'est la « communication numérique écrite » (ou « communication écrite en ligne ») - un hyper-genre sous-tendant des formes émergeant actuellement dans notre environnement verbal sur l'internet. Le volume est destiné aux étudiants en sciences de l'information et de la communication, en sciences politiques et en sciences du langage, et parait dans la collection Icom dirigée par Dominique Maingueneau.

On doit ce livre à un spécialiste du domaine, Michel Marcoccia, qui a déjà apporté beaucoup de contributions sur divers discours numériques : forums de discussion entre adolescents, courrier des lecteurs, forums des partis politiques et des entreprises, polylogues en ligne, etc. Ce chercheur définit cette forme de communication dès son introduction (pp. 7-I2) : «Cette dénomination renvoie aux nombreuses productions écrites réalisées par le moyen de l'internet ou de la téléphonie mobile » et contient « le courrier électronique, le forum de discussion, la liste de diffusion, le tchat, la messagerie instantanée, le SMS (ou texto), le blog, les plateformes de réseaux sociaux » (p.7). Des formes de la communication verbale qui interpellent fortement la linguistique générale et la linguistique du discours.

Le livre s'organise en trois parties, destinées à décrire les dispositifs utilisés, les spécificités des écrits numériques (essentiellement conversationnels et « contextualisés», p. II) et, enfin, quelques particularités pragmatiques (structuration et formats de participation, la construction des identités numériques et des relations interpersonnelles, etc.).

Chacune de ces parties est sous-divisée en chapitres, dans une démarche très systématique pour le lecteur, où sont également intégrés les apports des nombreux chercheurs ayant contribué à la description du domaine. Un sous-chapitre dresse un bref historique des dispositifs CMO (communication médiée par ordinateur) et CMT (communication médiée par téléphone) (pp. 17-21), passant en revue les technologies Minitel et les formes asynchrones et synchrones de communication (formes du courrier électronique, forums de discussion et listes de diffusion, pour les premières ; tchat et messagerie instantanée grand public, pour les secondes), pour arriver aux blogs, aux réseaux sociaux Facebook, Linkedln ou aux plateformes de wikis.

Un autre sous-chapitre explicite la profusion de termes utilisés pour dénommer ce type de communication non encore « stabilisé » dans l'esprit des utilisateurs ou dans la terminologie des chercheurs. Ainsi « électronique » et « numérique » se font-ils concurrence, en anglais ou en français, avec des dénominations comme: electronic communication, discours électronique médié, communication électronique scripturale, écriture numérique, écrits d'écrans, discours d'internet, activités technoscripturales, computer conversation, written interactive discours, écrit interactif et dialogique etc. (pp. 21-23), catégories quasi-synonymes. $\mathrm{Ce}$ côté terminologique s'avère intéressant pour le chercheur qui devra dorénavant s'habituer à utiliser de nouvelles catégories comme : persistance des messages (pp. 26-30), culture de « lulz » (Rire aux dépens d'autrui) (p. 33), flames (injures) (p. I34) pseudonymat, trolles (internaute postant sur des espaces de discussion des messages provocateurs) (p. 137), genre participatif (p. 139), identité numérique (p. 145), autonymes et avatars (p. 149) - toutes définies dans ce livre dès leurs apparitions dans le texte ; tout comme des abréviations nouvelles, telles CMDA (Computer-Mediated Discourse Analysis, p. 28), ASV (Âge, sexe, ville), pour la description de soi sur internet (p. 149). Nous ne mentionnons pas ici les abréviations déjà connues pour tout utilisateur d'internet et de messages électroniques.

Michel Marcoccia met en avant les avantages que peuvent tirer les chercheurs en linguistique de ces écrits sur internet, dont la taille (c'est un immense « corpus »!) et l'accessibilité permettent des reprises et des recherches beaucoup plus amples, une meilleure représentativité, sans parler du fait que la plupart s'offrent comme déjà transcrits par leurs producteurs (pp. 38, 39). Un problème qui reste pourtant non résolu est celui de la déontologie de ces reprises, et ce, de par la difficulté d'identifier si certains terrains sont publics ou privés (pp. 42-47). 
Mais un grand acquis, déjà constaté par la recherche linguistique, est la découverte de formes nouvelles, émergentes, non seulement au niveau du système de la langue (lexique, grammaire), mais également des « genres » nouveaux, pour la plupart hybrides. Or, ces formes ont récemment engendré un nouveau champ de recherche - une linguistique informatique et ce qu'il faut dorénavant appeler l'analyse des discours numériques (pp. 51-53) - proposant des catégories d'analyse innovantes pour décrire des formes discursives, adaptées au registre d'oral-écrit (ou graphique-parlé, langue orale scriptée, écrit oralisé, oral transcrit à la va-vite, selon les auteurs), à la plurisémioticité, au fort degré d'intertextualité et à leur dimension hypertextuelle (pp. 6|-7|).

Michel Marcoccia propose une analyse en cinq niveaux de ces formes discursives : typographique, orthographique, morphologique, lexical et syntaxique. Le chapitre consacré à cette analyse, qui passe en revue de nombreux apports récents dans le domaine pour différentes langues, oblige, dit l'auteur, à une remise en cause de l'affirmation de 200I de David Cristal selon qui, « le "langage numérique" (Netspeak) fonctionne comme une langue universelle » (p. 78). Plus détaillée est l'analyse du langage texto (SMS) (pp. 79-83).

Le livre attire l'attention sur ce que Dominique Maingueneau avait déjà appelé, en 2014, une « nouvelle textualité », avec les trois composantes de l'espace numérique : iconique, architecturale et procédurale (p. 88), composantes soutenues par divers logiciels. Sont soulignées les contributions essentielles de MarieAnne Paveau, qui propose les notions de « technologie discursive » et de « technodiscursivité », du fait de l'influence importante des outils technologiques sur ces nouvelles formes de communication qu'ils sont censés gérer. Car le mélange au message verbal des technosignes (commandes par mots-consignes, du type J'aime, répondre...) et des émoticônes change radicalement les modalités de composer les textes. Enfin, un autre linguiste (Alain Rabatel) a remarqué, dit Michel Marcoccia, un type particulier de citation, par lien, proposant de l'appeler citation potentielle ou à distance, ce qui fait de l'hypertextualité « la modalité la plus spécifiquement numérique de l'intertextualité », en réseau, avec des marqueurs spécifiques : liens, boutons, soulignements, variation de couleur - « signes passeurs 》, selon Julia Bonaccorsi (p. 105). Avec ces nouvelles possibilités, les discours se délinéarisent, les textes apparaissent comme instables et sans clôture, la lecture devient interactive et les écrits se conversationnalisent, allant de la forme privée vers la forme publique. C'est ce que Dominique Maingueneau, cité page I | |, appelle « conversécriture ».
Dans la dernière partie du livre, destinée à la description pragmatique de la communication écrite numérique, Michel Marcoccia montre comment des catégories comme médias de masse vs médias de communication interpersonnelle, les notions de public, semi-public et privé, celles de destinataires ratifiés et non ratifiés sont responsables de formes spécifiques d'échanges : certains tronqués, courts, intercalés, avec des interventions non adjacentes, ou thématiquement flous. Sous la problématique de la « performativité » des discours numériques, l'auteur range tous les moyens, essentiellement techniques, destinés à rentabiliser les messages : indicateurs de priorité (étiquette « urgence »), fonction « transférer », zone « sujet », émoticônes, etc. Il parle aussi d'autres fonctionnalités des systèmes, celles qui permettent la construction de communautés virtuelles plus ou moins stables, intéressantes aussi bien pour le linguiste que pour le sociolinguiste, déjà signalées en 1968 par John J. Gumperz qui les appelait « communautés en parole », génératrices de langages identitaires (p. 139). À la suite de différents « travaux empiriques », Michel Marcoccia décrit le phénomène comme dépendant de neuf conditions: sentiment d'appartenance, ritualisation de la parole, stabilisation de rôles dans le groupe, familiarité, réciprocité, partage des valeurs et des finalités, histoire conversationnelle commune, persistance des échanges, constitution d'une charte du groupe. La présentation de la collectivité par des marqueurs sociatifs et la présentation du soi par des marqueurs possessifs - terminologie reprise à Fanny Georges (Identités virtuelles. Les profils utilisateur du web 2.0., Paris, Éd. Questions théoriques, 2010) - deviennent de nouveaux terrains d'étude et, grâce à l'internet, beaucoup plus facilement investigables que les discours réels.

Michel Marcoccia conclut avec une remarque générale faisant référence aux rapports entre les hommes et les machines, et aux stratégies compensatoires auxquelles oblige ce binôme de nos jours. Nous croyons qu'un mérite particulier appartient aussi à l'éditeur, Armand Colin, pour sa politique de soutien aux publications universitaires et/ou d'éducation grand public : ce livre éclaire ce domaine de la communication numérique, qu'il faut non seulement comprendre et savoir utiliser, mais aussi savoir décrire.

Liana Pop

Université Babeş-Bolyai, RO-400084 liananegrutiu@yahoo.fr 